\title{
Anesthetic implications of the anomalous origin of coronary artery (AOCA)
}

\author{
Rahul Shabadi*, Nasser Al-Kemyani, Salman Siddiqui, Ramnarayan Rao and Tarek Alameddine \\ Department of Anesthesia, College of medicine and health sciences, Sultan Qaboos University, Oman
}

\begin{abstract}
This Article includes 2 case reports describing Anomalous Origin of the Coronary Artery (AOCA), the potentially fatal condition which warrants modification of anesthesia management. Our aim is to not only to highlight the potentially dangerous coronary anomalous origins, their association with sudden cardiac arrest but also addressing the anesthetic precautions and management plans for the patients with Abnormal Origin of Coronary Artery (AOCA).
\end{abstract}

\section{Case 1}

A 62 years female with previous history of bioprosthetic mitral valve replacement done 9 years back, presented with dyspnea on exertion. She had raised JVP and pulsatile hepatomegaly. On transthoracic echocardiography she was found to have severe leaking bioprosthetic valve causing severe mitral regurgitation. It was associated with tricuspid regurgitation and pulmonary hypertension. She had previous few admissions with cardiogenic shock and slow atrial fibrillation for which she was on tablet Warfarin and tablet Digoxin. Coronary angiography showed, Left Main Coronary artery (LMCA) originating aberrantly from the right coronary cusp almost very close to the Right Coronary artery (RCA) origin. LMCA was very long, wide and without any obstructive lesion bifurcating into Left Anterior descending (LAD) and Circumflex (CX). There were no major lesions in the coronaries. The Patient had no other comorbid conditions. The mitral valve replacement and tricuspid annuloplasty was proposed for the patient. The anesthesia Induction was done as per institutional protocol. Intra-operative transesophageal echocardiography revealed bioprosthetic mitral valve with severe mitral regurgitation. The Mean Gradient across Mitral valve was $3.7 \mathrm{~mm} \mathrm{Hg}$. The right and left atrium were dilated. Tricuspid valve showed severe regurgitation associated with hepatic venous flow reversal. Tricuspid annulus was $3.87 \mathrm{cms}$. Pulmonary systolic BP was $43-45 \mathrm{mmHg}$. Aortic and Pulmonary valves were normal. Rt. Coronary sinus of Valsalva giving rise to RCA and LMCA was seen well (Figure 1). Left ventricular function was 35$40 \%$. Inferior wall hyperkinesia was noted. Right ventricle was Grade II dilated. Mitral valve replacement with bioprosthetic valve size 29 $\mathrm{mm}$ was performed through trans-septal approach. Tricuspid valve annuloplasty with Carpenter Edward MC3 ring size $32 \mathrm{~mm}$ was done. Separation from CPB was done as per the protocol. Patient needed inotropes, Adrenaline $0.06 \mathrm{mcg} / \mathrm{kg} / \mathrm{min}$, Noradrenaline $0.06 \mathrm{mcg} / \mathrm{kg} /$ $\mathrm{min}$ and Dopamine $5 \mathrm{mcgs} / \mathrm{kg} / \mathrm{min}$. Patient was paced venticularlly for slow heart rate. After the completion of Inj. Protamine, the patient developed ventricular fibrillation. It was not responding to defibrillation so it was decided to reinitiate the CPB. Trial of separation from CPB was given after 20-25 min. of the rest. Inj. Amiodarone $300 \mathrm{mg}$ was given on pump before separation. Pt was paced venticularly for slow junctional rhythm. Patient again had recurrent ventricular fibrillation episodes at the chest closure. It responded to the internal defibrillation DC shocks. Pt was again put on CPB due to persistent ventricular arrhythmias. This time supported for $1.5 \mathrm{hr}$ on CPB. Inspite of all this efforts she had malignant arrhythmias after shifting to ICU. She didn't respond to medical management and patient's condition deteriorated continuously and on the next day patient expired in the ICU.

\section{Case 2}

19 years old male $166 \mathrm{~cm}$ tall and weighing $125 \mathrm{~kg}$ and his BMI was 45, a morbidly obese. He was known case of IDDM, adult Still's disease, Autoimmune hepatitis and hypertensive retinopathy. He had a previous admission for NSTMI. The patient was complaining of exertional chest

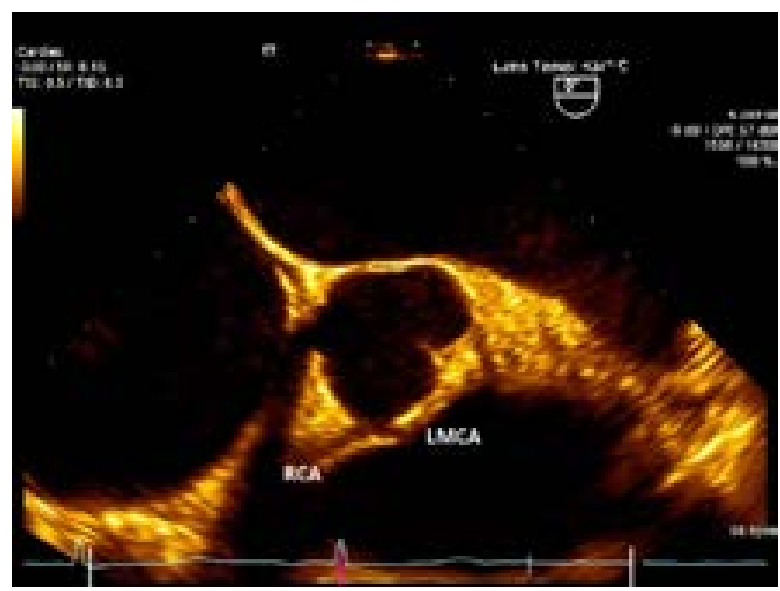

Figure 1. TEE image showing Left Main Coronary Artery (LMCA) originating from Right Sinus of Valsalva (Patient1).

Correspondence to: Dr. Rahul Shabadi, Department of Anesthesia, College of medicine and health sciences, Sultan Qaboos University, Oman; Tel: +96824144778 Extn: 4738; E-mail: drrahoolndrrahool@gmail.com

Key words: Abnormal Origin of Coronary artery (AOCA), sudden cardiac death, Anesthesia management

Received: June 03, 2016; Accepted: July 08, 2016; Published: July 13, 2016 
pain which was persistent since more than 6 month. It was associated with shortness of breath, palpitation and dizziness. These symptoms were present mainly on exertion. His METs were estimated as 4-5. He was not smoker. He had a strong family history of IHD in mother. The Cardiac CT showed the anomalous right coronary artery arising from the left coronary sinus of Valsalva. RCA was dominant and located anterosuperior to the origin of the LMCA. There was narrowing of the proximal segment and narrowing of the ostium and coursing between the root of the aorta and the main pulmonary artery. LM, LAD, LCX and their branches were normal. Myocardial perfusion study was normal. Tran-thoracic echocardiography was normal with ejection fraction of $60 \%$. The induction of anesthesia was done as per institutional protocol. Intraoperative trans-esophageal echocardiography was performed. It revealed left ventricular hypertrophy and good function. Mitral valve showed mild regurgitation. Aortic valve was tri-leaflet. The LMCA origin seen well and Right Coronary Artery origin seen near to LM origin (Figure 2). Right Ventricular function was good and other valves were normal. On Pump De-roofing of right Coronary Ostia was done. Separation from CPB was done without inotropes. Postsurgical repair transesophageal echocardiography showed the flow through right coronary ostium and the nonturbulent flow through the RCA. There was absence of new regional wall motion abnormality of left ventricle. The patient's recovery was uneventful in ICU and was discharged home on $7^{\text {th }}$ post-operative day.

\section{Discussion}

Congenital anomalies of the coronary arteries officially reported to occur in $0.6 \%-1.3 \%$ of the general population [1]. Coronary artery anomalies can occur in association with other congenital heart defects or as an isolated abnormality. The clinical presentation and severity of coronary artery anomalies varies considerably. Certain coronary artery anomalies are found incidentally, and are clinically silent. But at the other end of the disease spectrum are anomalies which may present clinically. They present with angina, dyspnea, and syncope as happened with our second case and in severe cases arrhythmias, myocardial infarction, and sudden death [2,3] After the Hypertrophic cardiomyopathy, the anomalous origin of coronary arteries (AOCA) is the most common causes of sudden cardiac death in young adults in the USA. It is found in $13 \%$ of autopsies in post mortem cases [4,5]. Of many subtypes of this anomaly two most important subtypes are those in which the left main coronary artery originates from the right sinus of Valsalva (ALMCA) or the right coronary artery originates from

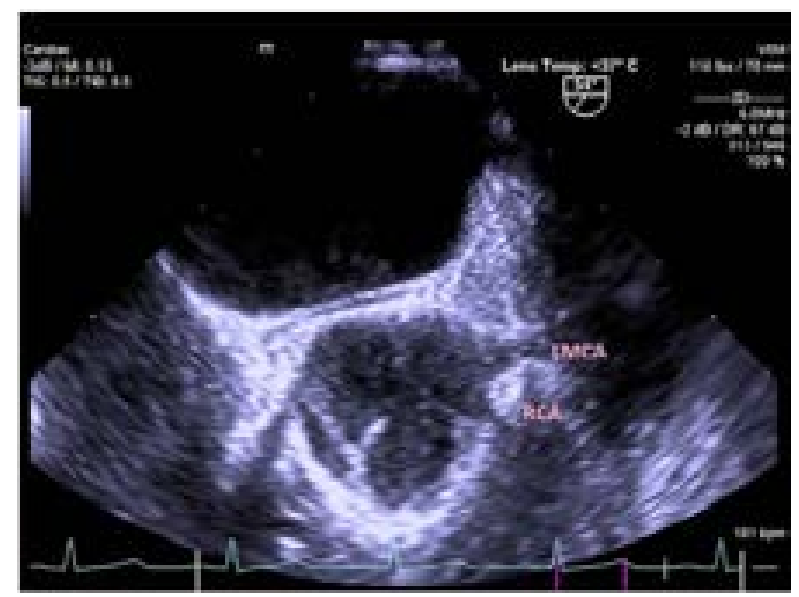

Figure 2. TEE showing Right Coronary Artery originating from Left Coronary sinus of Valsalva (Patient 2).

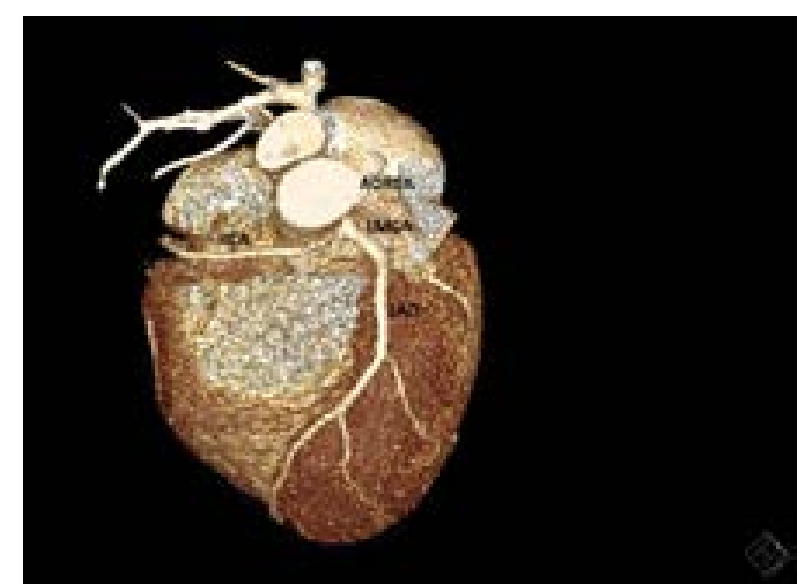

Figure 3. CardiacCT SCAN image showing Right Coronary Artery originating from Left Coronary sinus of Valsalva (Patient 2).

the left sinus of Valsalva (ARCA). In a majority of cases intramural course of the anomalous coronary artery is found, it traverses within the aortic wall, between the aorta and pulmonary artery, then leaves the aortic wall from the correct sinus. Both of the aforementioned defects (ALMCA and ARCA), particularly those have an intramural course, have been associated with sudden cardiac death [4].

Sudden death occurs in these patients because of a reduction in blood flow in the anomalous vessel, resulting in myocardial ischemia or infarction [6]. The pathophysiology is due to reduced coronary blood flow in these patients. Due to acute take off of the anomalous coronary artery often takes, the ostium of the anomalous artery is slit-like, rather than round as in its normal counterpart. During systole this slit-like orifice gets further compressed, which may occlude further blood flow. Also proposed theory is that kinking or torsion of the anomalous artery may occur as it travels between the great vessels. Another hypothesis is the intramural path within the aortic wall, and can be is a potentially lethal condition due to compression of the coronary artery which might occur during times of increased aortic pressure, like during exercise. Most likely multifactorial effect may precipitates myocardial ischemia, infarction, and sudden cardiac death in cases of AOCA. Amongst them the most important being, the increased in aortic wall tension and the sizes of Aortic and Pulmonary roots, leading to compression or kinking of abnormal course of coronary. The patients diagnosed with ALMCA or ARCA, should be immediately considered for the treatment. Though medical management is an option, surgery remains the most widely used [6-10]. At present the general agreement among most physicians is, if a patient is symptomatic or shows evidence of ischemia in the presence of an ALMCA or ARCA, surgery should be offered, particularly below of 30 years of the age $[4,10]$. Most worrying surgery in this patient subtype would be aortic valve replacement. These patients especially with intramural course can have worsening of the condition because of the extrinsic compression by prosthetic ring [11]. AOCA has been linked with genetic association. Family history of sudden cardiac death or history suggestive of angina in absence of risk factors of myocardial infarction especially in young patient should raise the suspicion AOCA [12].

The anesthetic experiences about the AOCA and its management are limited. Most of them are mainly from cardiac surgery and cardiology literatures. An anesthesiologist has to be cautious from preoperative evaluation of the patient till the post- operative period, as these patients are at very high risk of sudden cardiac death during induction of anesthesia [5]. Sudden cardiac death under anesthesia 
is associated with arrhythmogenic right ventricular cardiomyopathy, myocardial diseases, fibrosis of the Bundle of Hiss, AOCA were the most frequently found diseases. The cases where an anomalous origin or route of the coronary artery was identified, can have circulatory arrest during induction of anesthesia [5]. Preoperative evaluation of heart function with ECG, echocardiography and intraoperative transesophageal echocardiography will play pivotal role in patient management. These patients may present with non ST MIs, as this second case was presented or the patient can still have ongoing ischemia The factors which will increase rate pressure product and eventually oxygen demand of the heart can lead to untoward event.

The emphasize will be to maintenance of hemodynamic stability (avoiding hypotension, hypertension, and tachycardia) and the optimization of oxygen-carrying capacity (avoiding hypoxia and anemia), to preserve the fine balance between myocardial oxygen supply and demand. So during Induction, the extra caution to be given to maintain the heart rate $60-80$ beats $/ \mathrm{min}$ and blood pressure with $30 \%$ of the baseline value. Taking into consideration of the compression effects from aorta and pulmonary trunk, the factors which will cause distension of aorta or pulmonary trunk should be avoided. This includes preoperative strenuous exercise or exertion, rapid transfusion of the fluids, hypoxia, and hypercarbia [12]. Intraoperative 5 lead ECG for monitoring of ST segment, invasive arterial and central venous pressure monitoring will give close idea about the hemodynamics. The fluid overload can be easily avoided by CVP monitoring and transesophageal echocardiography. Intraoperative transesophageal echocardiography helps to find out the course of the coronary artery. It helps us to monitor contractility, new RWMA, left ventricular filling and preload. The Most important is, it is the first tool which will give an idea about surgical repair and its outcome in patients who are undergoing surgical intervention for coronary artery. The nonturbulent flow in the coronaries, absence of new RWMA, presence of good contractility of the heart can give fair idea about the surgical repair. The presence of New RWMA or poor LV function, increasing requirements of the Inotropes, absent laminar flow, presence turbulent flow in the coronary ostia or coronaries should be considered as alarming signs.

\section{Conclusion}

The patents diagnosed with Abnormal Origin of Coronary origin should be dealt with extra precautions, as the risk of sudden cardiac arrests are high. The anesthesiologist should explain this risk to the patient and family well. This kind of anomaly warrants modification of anesthesia management plan and more close cardiac monitoring. Transesophageal echocardiography can be very useful tool to monitor these patients, especially who come for surgical intervention for coronary arteries.

\section{References}

1. Liberthson RR,Dinsmore RE,Bharti S, Rubestein JJ, Caulfield J, Wheeler ED (1974) Aberrant coronary artery origin from the aorta: diagnosis and clinical significance. Circulation 50: 774-779. [Crossref]

2. Liberthson RR (1996) Sudden death from cardiac cuases in children and young adults N Engl J Med 334:1039-1044. [Crossref]

3. Maron BJ, Shirani J, Poliac LC, Mathenge R, Roberts WC, Mueller FO (1996) Sudden death in young competitive athletes: clinical, demographic, and pathological profiles. JAMA 276:199-204. [Crossref]

4. Basso C, Maron BJ, Corrado D, Thiene G (2000) Clinical profile of congenital coronary artery anomalies with origin from the wrong aortic sinus leading to sudden death in young competitive athletes. J Am Coll Cardiol 35: 1493-1501. [Crossref]

5. Arya VK, Bangaari A, Rajeev S, Sharma A, Minz M, Rohit MK (2009) Intraoperative myocardial ischemia during renal transplantation caused by anomalous origin of the right coronary artery. Annulus of Cardiac Anesthesia 12: 57-62. [Crossref]

6. Davis JA, Cecchin F, Jones TK, Portman MA (2001) Major coronary artery anomalies in a pediatric population: incidence and clinical importance. J Am Coll Cardiol 37 593-597. [Crossref]

7. Cohen AJ, Grishkin BA, Helsel RA, Head HD (1989)Surgical therapy in the management of coronary anomalies: emphasis on utility of internal mammary artery grafts. Ann Thorac Surg 47:630-637. [Crossref]

8. Frommelt PC, Frommelt MA, Tweddell JS, Jaquiss RD (2003) Prospective Echocardiographic diagnosis and surgical repair of Anomalous origin of a coronary artery from the opposite sinus with an interarterial course. J Am Coll Cardiol 42: 148154. [Crossref]

9. Romp RL, Herlong RL, Landolfo CK, Sander SP, Miller CE, Ungerleider RM, Jaggers $\mathrm{J}$ (2003) Outcome of unroofing procedure for repair of anomalous aortic origin of left or right coronary artery. Ann Thorac Surg 76: 589-596. [Crossref]

10. Lipsett J, Cohle SD, Berry PJ, Russell G, Byard RW (1994)Anomalous coronary arteries: a multicenter pediatric autopsy study. Pediatr Pathol 14:287-300. [Crossref]

11. Angelini P (2007) Coronary Artery Anamolies:An entity in search of an Identity Circulation 115:1296-1305. [Crossref]

12. Daher M, Zanatta AR, Henz BD, da Silva MC, dos Santos SN, Leite LR (2012) Sudden cardiac arrest in general anesthesia as the first manifestation of anomalous origin of the left coronary artery. Rev. Bras. Anestesiol 62:878-884. [Crossref]

Copyright: (C2016 Shabadi R. This is an open-access article distributed under the terms of the Creative Commons Attribution License, which permits unrestricted use, distribution, and reproduction in any medium, provided the original author and source are credited. 